\title{
Valproato de sódio: efeitos colaterais em crianças
}

\author{
Valproate therapy: side effects in children
}

\section{Marlene Pereira Garanito ${ }^{1}$, Cynthia Rothschild ${ }^{2}$, Fernanda Glaucia L. Camlofski ${ }^{3}$, Edi Cabral ${ }^{4}$}

\section{RESUMO}

Objetivo: $\mathrm{O}$ valproato de sódio é um fármaco anticonvulsivante usado com frequência no manejo dos quadros epilépticos refratários na infância e habitualmente apresenta boas taxas de resposta e toxicidade aceitável. O objetivo deste artigo é destacar os efeitos colaterais secundários ao uso prolongado de valproato de sódio em uma criança e alertar para a importância do acompanhamento dos pacientes em uso crônico de anticonvulsivantes.

Descrição do caso: Criança do sexo masculino, com quatro anos e oito meses, portadora de síndrome de West, em uso de valproato de sódio desde os seis meses de idade, admitida no hospital com anemia, macrocitose, plaquetopenia, deficiência de vitamina B12, hipoalbuminemia e hiponatremia. Durante a internação e o acompanhamento ambulatorial, relacionou-se o quadro clínico ao uso do valproato de sódio.

Comentários: A toxicidade hematológica do valproato de sódio é bem conhecida e comum, podendo variar em relação à época de instalação e gravidade. Os achados mais frequentes são a plaquetopenia e a macrocitose. Além das alterações hematológicas, a literatura relata outros efeitos colaterais relacionados ao uso do fármaco. Destaca-se a importância do conhecimento e monitorização dos eventos adversos nos pacientes submetidos a esse tipo de terapia, para que eles possam ser detectados e tratados o mais precocemente.

Palavras-chave: ácido valpróico; convulsão; criança.

\section{ABSTRACT}

Objective: Valproic acid is an anticonvulsant frequently used in the management of childhood refractory epilepsy with good clinical responses and acceptable toxicity. The objective of this case report is to describe side effects associated with long-term valproate therapy in a child and to warn about the importance of monitoring patients in chronic use of anticonvulsants.

Case description: This male child, four years and eight months old, with West syndrome, had been using sodium valproate since six months of age. The patient was admitted with anemia, macrocytosis, thrombocytopenia, deficiency of vitamin B12, hypoalbuminemia and hyponatremia. The clinical signs were associated to chronic sodium valproate use.

Comments: Sodium valproate hematologic toxicity is frequent, varying in onset and severity. The most common findings are thrombocytopenia and macrocytosis. In addition to the hematological toxicity, literature reports other side effects associated with valproic acid therapy. It is important to know and to monitor adverse effects in patients undergoing sodium valproate therapy in order to detect and treat them as early as possible.

Key-words: valproic acid; seizure; child.
Instituição: Universidade de Santo Amaro (Unisa) e Hospital Geral do Grajaú (HGG), sob gestão da Associação Congregação de Santa Catarina, São Paulo, SP, Brasil

'Médica Hematologista Pediatra do Ambulatório Médico de Especialidades Interlagos (AME-Interlagos) do Hospital Geral Grajaú (HGG) e da Faculdade de Medicina da Unisa, São Paulo, SP, Brasil

${ }^{2}$ Médica Hematologista do AME - Interlagos do HGG, São Paulo, SP, Brasil

${ }^{3}$ Residente de Pediatria da Unisa, São Paulo, SP, Brasil

${ }^{4}$ Chefe da Disciplina de Hematologia e Banco de Sangue da Unisa, São Paulo, SP, Brasil
Endereço para correspondência:

Marlene Pereira Garanito

Rua Venâncio Aires, 315, apto. 71

CEP 05024-030 - São Paulo/SP

E-mail: marlene.garanito@ig.com.br

Fonte financiadora: Centro de Ensino e Pesquisa Prof. Dr. Liberato John Alphonse Di Dio (Cenepes)

Recebido em: 25/11/08 Aprovado em: 26/3/09 


\section{Introdução}

O valproato de sódio é o fármaco de escolha para o tratamento de pacientes portadores de crises epilépticas focais ou generalizadas, além de outras síndromes epileptiformes, como a síndrome de $\mathrm{West}^{(1,2)}$. Atualmente, vários estudos vêm sendo realizados com o objetivo de tentar ampliar o uso desse fármaco no tratamento de outras doenças, como o transtorno bipolar, as alterações psiquiátricas relacionadas ao abuso de álcool e cocaína, e a doença de Alzheimer; mais recentemente, pesquisa-se o uso do valproato de sódio por seu efeito antitumoral ${ }^{(3)}$.

Embora habitualmente seja bem tolerado, mais atenção tem sido dada aos efeitos colaterais já descritos, uma vez que é um dos anticonvulsivantes mais utilizados mundialmente e em larga escala. Dessa forma, as alterações desencadeadas pelo uso da droga em questão começam a ser melhor diagnosticadas e mais precocemente tratadas pelos especialistas.

Os efeitos adversos associados ao uso do valproato de sódio, independentemente do tempo de uso, podem ou ser leves e reversíveis ou graves, irreversíveis e fatais ${ }^{(1)}$, incluindo desde queda de cabelo, náuseas e vômitos, até teratogenicidade, toxicidade hepática severa, alteração do turnover ósseo e discrasias sanguíneas ${ }^{(4)}$.

A maioria dos relatos de casos publicados na literatura se refere a pacientes com um único efeito colateral do fármaco. Raros são os artigos que descrevem vários efeitos adversos, secundários ao uso do valproato de sódio em um mesmo paciente. Desta forma, o objetivo deste artigo é destacar os vários efeitos colaterais secundários ao uso prolongado do valproato de sódio, observados em uma criança, e alertar para a importância do acompanhamento de pacientes em uso crônico do medicamento.

\section{Descrição do caso}

Paciente com quatro anos e oito meses, sexo masculino, branco, em acompanhamento no ambulatório de Neurologia desde os seis meses de idade com o diagnóstico de síndrome de West e, desde então, em uso de clobazam $(0,5 \mathrm{mg} / \mathrm{kg} / \mathrm{dia})$ e valproato de sódio $(90 \mathrm{mg} / \mathrm{kg} / \mathrm{dia})$, mantendo-se nível sérico adequado dos dois anticonvulsivantes.

O paciente foi admitido no Pronto Socorro Infantil devido a um quadro de broncoespasmo e infecção de vias aéreas superiores. $\mathrm{Na}$ ocasião, os exames evidenciaram anemia normocítica e normocrômica, plaquetopenia e deficiência de vitamina B12, sendo normais todos os outros exames: coagulograma, bilirrubinas, desidrogenase lática, dosagem de ácido fólico e ferritina (Tabela 1, primeira internação). O mielograma evidenciou a presença de megacariócitos normais e padrão megaloblástico das outras séries (Figuras 1 e 2). Do ponto de vista hematológico, o quadro foi interpretado como anemia secundária à deficiência de vitamina B12 associada à trombocitopenia imune, uma vez que a plaquetopenia evoluiu com melhora espontânea, antes mesmo da administração de vitamina B12. Com relação ao tratamento, foi realizada

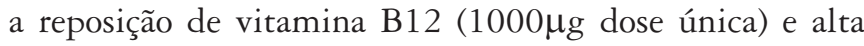
hospitalar para acompanhamento ambulatorial.

Após um mês, o paciente foi reinternado devido a novo quadro de infecção de vias aéreas superiores associado a petéquias na face e tórax e edema de membros inferiores. Apresentava macrocitose e plaquetopenia, hiponatremia, hipoalbuminemia, osmolaridade e sódio urinário baixos e elevação de aspartato aminotransferase, com o restante dos exames normais: alanina aminotransferase, uréia, creatinina,

Tabela 1 - Resultados de exames laboratoriais

\begin{tabular}{lcccc}
\hline & $\begin{array}{c}\text { Primeira } \\
\text { internação }\end{array}$ & $\begin{array}{c}\text { Segunda } \\
\text { internação }\end{array}$ & Ambulatório & Referência \\
\hline $\mathrm{Hb}(\mathrm{g} / \mathrm{dL})$ & 9 & 12 & 12 & 12,5 \\
$\mathrm{Hto}(\%)$ & 26 & 34 & 35 & 37 \\
$\mathrm{VCM}(\mathrm{fL})$ & 90 & 100 & 100 & 81 \\
$\mathrm{CHCM}(\mathrm{g} / \mathrm{dL})$ & 30 & 34 & 35 & 34 \\
Leucócitos $\left(\mathrm{mm}^{3}\right)$ & 5.400 & 5.200 & 6.500 & $5-10.000$ \\
Plaquetas $\left(\mathrm{mm}^{3}\right)$ & 15.000 & 18.000 & 201.000 & $150-400.000$ \\
Vitamina B12 $(\mathrm{pg} / \mathrm{mL})$ & 157 & - & 505 & $190-900$ \\
Sódio $(\mathrm{mEq} / \mathrm{L})$ & 125 & 129 & - & $135-145$ \\
Sódio urina $(\mathrm{mEq} / \mathrm{L})$ & - & 113 & - & $116-270$ \\
Osmolaridade urina $(\mathrm{mOsm} / \mathrm{L})$ & - & 48,9 & - & $656-984$ \\
Albumina $(\mathrm{g} / \mathrm{dL})$ & - & 2,6 & 3,4 & $3,8-5,0$ \\
TGO $(\mathrm{Ul} / \mathrm{L})$ & - & 80 & - & Até 37 \\
Outros & Normais* & Normais** & - & - \\
\hline
\end{tabular}

${ }^{*}$ Coagulograma, bilirrubinas, DHL, ferritina, dosagem de ácido fólico; **potássio, creatinina, TGP, urina I, proteinúria de 24 horas. 


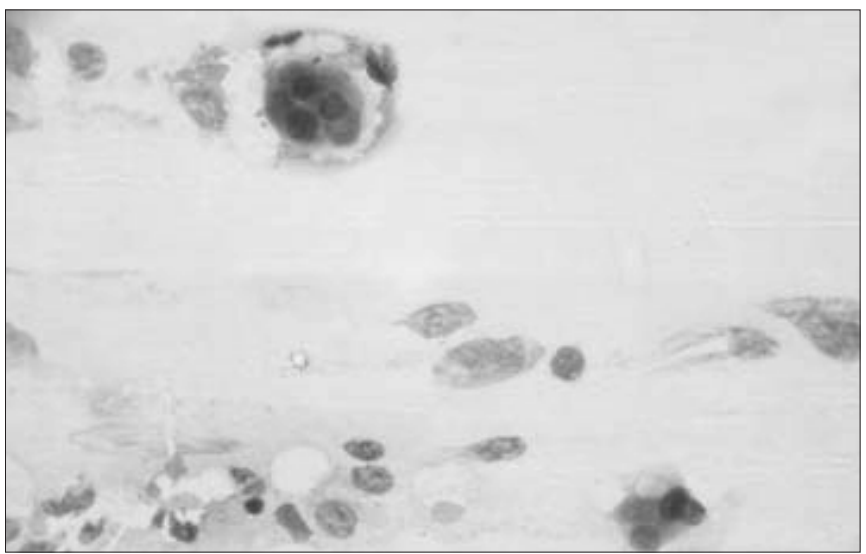

Figura 1 - Megacariócito (Leishman).

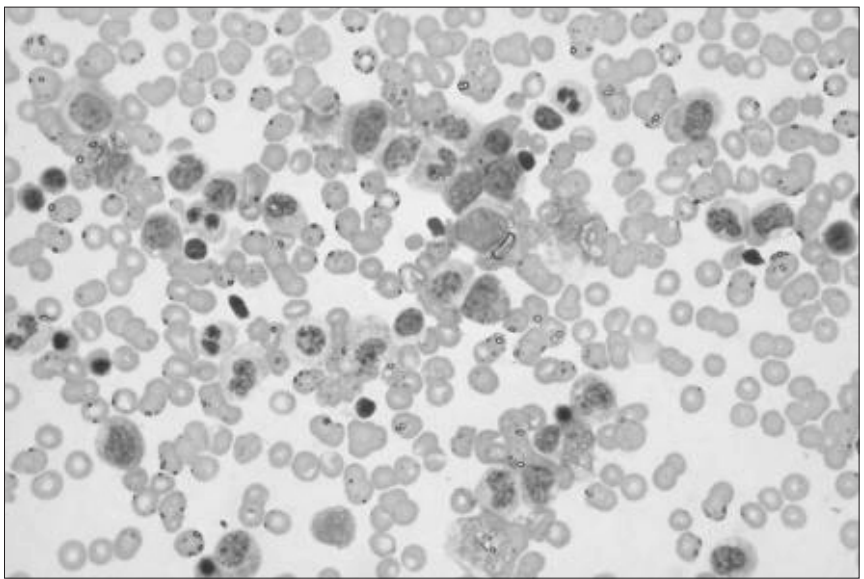

Figura 2 - Medula óssea com aspecto megaloblastoide

(Leishman).

urina tipo I e proteinúria de 24 horas (Tabela 1, segunda internação). Houve melhora parcial da plaquetopenia (83.000/ $\mathrm{mm}^{3}$ ) após três doses de metilprednisolona $(30 \mathrm{mg} / \mathrm{kg} / \mathrm{dia})$. A hiponatremia foi considerada dilucional. Este paciente recebeu reposição de albumina uma vez, com melhora parcial do nível sérico e do quadro clínico. Com relação à hiponatremia, optou-se por conduta expectante, uma vez que o paciente se apresentava assintomático. A criança recebeu alta para acompanhamento nos ambulatórios de Neurologia e Hematologia.

Durante o acompanhamento ambulatorial, o paciente manteve a hipoalbuminemia, a hiponatremia e a macrocitose, apesar da correção dos níveis de vitamina B12; além disso, não voltou a apresentar citopenias (Tabela 1 : ambulatório). Com base em dados da literatura, o valproato de sódio foi substituído por topiramato, ocorrendo posteriormente a resolução da macrocitose, hipoalbuminemia e hiponatremia.
Atualmente, o paciente está em uso de topiramato, clobazam e clonazepam, sem reposição de complexos vitamínicos, e mantém os achados do hemograma e o nível sérico de albumina e sódio normais.

\section{Discussão}

Uma das grandes discussões quanto ao uso dos anticonvulsivantes são os seus potenciais efeitos colaterais, motivo este de grande preocupação e de consultas frequentes aos médicos hematologistas. Nesse grupo de fármacos, destacase o valproato de sódio, usado habitualmente no controle de quadros de epilepsia refratária da infância, com boa eficácia clínica ${ }^{(1)}$.

Do ponto de vista hematológico, este fármaco pode causar depressão da medula óssea por ação direta, levando à aplasia medular ou à síndrome mielodisplásica - que pode resultar em citopenia periférica, afetando uma ou mais linhagens celulares -, observando-se, portanto, plaquetopenia, neutropenia e/ou macrocitose eritrocitária com ou sem anemia ${ }^{(1,5)}$. A plaquetopenia é o efeito colateral mais frequente, com incidência entre 5 e $60 \%$ dos indivíduos que tomam o medicamento. $\mathrm{O}$ intervalo de tempo entre o início do uso do valproato de sódio e a sua detecção é variável (de oito dias a 18 meses). A plaquetopenia se caracteriza por ser dose dependente, com apresentação clínica que varia desde quadros leves até graves. A sua gênese parece se dever à depressão direta da medula óssea por elevados níveis de valproato de sódio (maiores que $40 \mathrm{mg} / \mathrm{kg} / \mathrm{dia}$ ) ou pode ser secundária à destruição imune-mediada, devido à semelhança entre os componentes do valproato de sódio e as membranas celulares. Habitualmente, o quadro é reversível com a redução da dose do medicamento ou sua suspensão ${ }^{(4-12)}$. No caso apresentado, o estudo da medula óssea evidenciou a presença de megacariócitos normais e houve resposta satisfatória após o uso de corticoesteróides, confirmando-se o diagnóstico de plaquetopenia de origem imunológica.

No que se refere à macrocitose e à deficiência de vitamina B12 associadas ao uso de anticonvulsivantes, a literatura ainda é controversa. Ao contrário dos níveis séricos de folato, que sabidamente se apresentam reduzidos nos pacientes em uso desta medicação, os níveis séricos de vitamina B12 podem estar reduzidos, normais ou aumentados em pacientes com epilepsia e em uso de valproato de sódio ${ }^{(13)}$. Alguns estudos relatam a presença de macrocitose associada ao valproato de sódio sem que seja observada deficiência de ácido fólico ou vitamina B12. O mecanismo envolvido ainda não está claro. 
Segundo alguns autores, altas concentrações de valproato de sódio induziriam alterações na membrana fosfolipídica do eritrócito, responsáveis pela macrocitose $e^{(14,15)}$. No caso relatado, o paciente apresentou deficiência de vitamina B12 e macrocitose com padrão megaloblástico em medula óssea e sem história de perdas ou dieta inadequada que pudessem justificar a deficiência de tal vitamina. Não foram realizados testes para avaliar a absorção de vitamina B12. A carência vitamínica foi corrigida, mas houve a persistência da macrocitose, apesar de níveis séricos normais de vitamina B12 e ácido fólico. A anemia megaloblástica só foi resolvida após a retirada do valproato de sódio, vindo a confirmar a associação entre o uso do medicamento e a macrocitose.

Outros efeitos colaterais atualmente descritos são a hipoalbuminemia e a hiponatremia associadas à síndrome de secreção inapropriada de hormônio antidiurético-like $(\text { SIADH-like })^{(1,16-19)}$. Uma das hipóteses para a ocorrência da SIADH-like é um efeito direto do valproato de sódio na função das células tubulares renais ${ }^{(20)}$.

O valproato de sódio é metabolizado quase exclusivamente no fígado e, como consequência, existe boa documentação de efeitos colaterais potencialmente graves, incluindo a falência hepática. Além disso, outros efeitos colaterais observados são resultantes da capacidade do valproato de sódio de inibir a secreção vesicular e reduzir a síntese de proteínas plasmáticas, incluindo a albumina ${ }^{(19)}$. Essa hepatotoxicidade relacionada ao uso de valproato de sódio pode ser reversível ou não ${ }^{(18)}$. No caso relatado, o paciente apresentava exames de urina tipo I (sedimento quantitativo) e proteinúria de 24 horas normais, fatos que excluíam a perda de proteína pela urina que justificasse a hipoalbuminemia. Quanto à função hepática, a criança apresentava coagulograma normal e um aumento discreto de aspartato aminotransferase, o que também não justificava o quadro clínico. Não ocorreram mudanças na dieta desse paciente, que se encontrava com peso e estatura adequados para a idade. Assim, a hipoabuminemia não poderia ser justificada por alterações nutricionais, renais ou hepáticas. Após a retirada do valproato de sódio, houve a normalização do nível sérico de albumina e reversão do quadro clínico. Ainda não está totalmente esclarecido o mecanismo envolvido na gênese da hipoalbuminemia mediada pelo valproato de sódio ${ }^{(19)}$.

Associado ao quadro de hipoalbuminemia, este paciente apresentou hiponatremia dilucional assintomática, que também se resolveu após a suspensão do medicamento. Contudo, vale destacar a associação descrita entre o uso de valproato de sódio e a SIADH-like, cursando com hiponatremia, concentração de sódio urinário normal e osmolaridade urinária aumentada, reversível após a suspensão do medicamento ${ }^{(16)}$.

Assim, o caso raro de uma criança, aqui relatado, com vários efeitos colaterais simultâneos secundários ao uso prolongado de valproato de sódio, destaca a importância do conhecimento e monitorização de pacientes submetidos a esse tipo de terapia, a fim de que esses efeitos colaterais possam ser detectados e tratados o mais precocemente possível, evitando-se repercussões clínicas indesejáveis.

Finalmente, vale ressaltar que a literatura ainda desconhece o motivo pelo qual a maioria dos pacientes em uso de valproato de sódio não apresenta efeito colateral algum, enquanto outros apresentam uma grande variedade de efeitos simultâneos, com diferentes mecanismos envolvidos e relatados ${ }^{(2)}$.

\section{Referências bibliográficas}

1. Acharya S, Bussel JB. Hematologic toxicity of sodium valproate. J Pediatr Hematol Oncol 2000;22:62-5.

2. Gerstner T, Bauer MO, Longin E, Bell N, Koenig SA. Reversible hepatotoxicity, pancreatitis, coagulation disorder and simultaneous bone marrow suppression with valproate in a 2-year-old girl. Seizure 2007;16:554-6.

3. Goto S, Seo T, Hagiwara T, Ueda K, Yamauchi T, Nagata S et al. Potential relationships between transaminase abnormality and valproic acid clearance or serum carnitine concentrations in Japanese epileptic patients. J Pharm Pharmacol 2008;60:267-72.

4. Cannizzaro E, Albisetti M, Wohlrab G, Schmugge M. Severe bleeding complications during antiepileptic treatment with valproic acid in children. Neuropediatrics 2007;38:42-5.

5. Ganick DJ, Sunder T, Finley JL. Severe hematologic toxicity of valproic acid. A report of four patients. Am J Pediatr Hematol Oncol 1990;12:80-5.

6. Nasreddine W, Beydoun A. Valproate-induced thrombocytopenia: a prospective monotherapy study. Epilepsia 2008;49:438-45.
7. Finsterer J, Pelzl G, Hess B. Severe, isolated thrombocytopenia under polytherapy with carbamazepine and valproate. Psychiatry Clin Neurosci 2001;55:423-5.

8. Delgado MR, Riela AR, Mills J, Browne R, Roach ES. Thrombocytopenia secondary to high valproate levels in children with epilepsy. J Child Neurol 1994;9:311-4.

9. $\mathrm{Ko} \mathrm{CH}$, Kong CK, Tse PW. Valproic acid and thrombocytopenia: cross-sectional study. Hong Kong Med J 2001;7:15-21.

10. Barr RD, Copeland AS, Stockwell ML, Morris N, Kelton JC. Valproic acid and immune thrombocytopenia. Arch Dis Child 1982;57:681-4.

11. Brichard B, Vermylen C, Scheiff JM, Ninane J, Cornu G. Haematological disturbances during long-term valproate therapy. Eur J Pediatr 1994;153: 378-80.

12. Gerstner T, Teich M, Bell N, Longin E, Dempfle CE, Brand J et al. Valproateassociated coagulopathies are frequent and variable in children. Epilepsia 2006;47:1136-43. 
13. Karabiber H, Sonmezgoz E, Ozerol E, Yakinci C, Otlu B, Yologlu S. Effects of valproate and carbamazepine on serum levels of homocysteine, vitamin B12 and folic acid. Brain Dev 2003; 25:113-5.

14. Tsivkin R, Saito E, Kafantaris V. Macrocytosis associated with divalproex treatment. J Child Adolesc Psychopharmacol 2005;15:1011-4.

15. Ozkara C, Dreifuss FE, Apperson Hansen C. Changes in red blood cells with valproate therapy. Acta Neurol Scand 1993;88:210-2.

16. Ikeda K, Moriyasu H, Yasaka M, Oita J, Yamaguchi T. Valproate related syndrome of inappropriate secretion of antidiuretic hormone (SIADH) - a case report. Rinsho Shinkeigaku 1994;34:911-3.
17. Branten AJ, Wetzels JF, Weber AM, Koene RA. Hyponatremia due to sodium valproate. Ann Neurol 1998;43:265-7.

18. Koenig SA, Buesing D, Longin E, Oehring R, Häussermann P, Kluger G et al. Valproic acid-induced hepatopathy: nine new fatalities in Germany from 1994 to 2003. Epilepsia 2006;47:2027-31.

19. Rugino TA, Janvier YM, Baunach JM, Bilat CA. Hypoalbuminemia with valproic acid administration. Pediatr Neurol 2003;29:440-4.

20. Bavbek N, Alkan R, Uz E, Kaftan O, Akcay A. Hyponatremia associated with sodium valproate in a 22-year-old male. Nephrol Dial Transplant 2008; 23:410. 